\title{
Reconsidering the relationship between virally induced bacterial mortality and frequency of infected cells
}

\author{
Brian Binder* \\ Department of Marine Sciences, University of Georgia, Athens, Georgia 30602-3636, USA
}

\begin{abstract}
The relative contribution of viral lysis to overall mortality in aquatic bacterial populations is often estimated as twice the frequency of infected cells (FIC). The 'factor-of-two rule' upon which this estimate is based assumes (1) steady-state conditions, (2) that latent period is equivalent to generation time, and (3) that infected cells are not grazed. FIC values for this calculation are themselves derived from measurements of the frequency of visibly infected cells (FVIC) by the use of a simple conversion factor. A steady-state model was developed to more rigorously define the relationships between FlC, FVIC, and the fraction of mortality from viral lysis (FMVL). This model shows that even under the restrictive assumptions listed above, the factor-of-two rule systematically overestimates FMVL for typically reported values of FVIC. The model also shows that although grazing on infected cells further reduces FMVL for a given estimate of FIC, at the same time such grazing increases FIC for a given measurement of FVIC. In combination, these 2 effects minimize the influence of grazing on the calculation of FMVL from FVIC. Overall, the relationship between FMVL and FVIC is well approximated as follows: FMVL $\equiv F V I C /[\gamma \ln (2)(1-\varepsilon-F V I C)]$, where $\gamma=$ the ratio between the latent period and generation time, and $\varepsilon=$ the fraction of the latent period during which viral particles are not yet visible. Using typically observed values of FVIC, and assuming that $\gamma=1$ (per assumption 2, above) and $\varepsilon=0.186$ (per literature estimates), the model suggests that, on average, viral lysis accounts for approximately $22 \%$ (range: 4.5 to $45 \%$ ) of total bacterial mortality in a range of aquatic environments, corresponding to a mean overestimate of $24 \%$ (range: 4 to $44 \%$ ) by the factor-of-two rule. Perhaps most importantly, the model shows that calculations of FMVL from FIC or FVIC are very sensitive to changes in the relative length of the latent period $(\gamma)$ and in the assumed proportion of the latent period during which viral particles are not recognizable $(\varepsilon)$. Constraining these 2 factors would greatly improve the reliability of FMVL calculations.
\end{abstract}

KEY WORDS: Aquatic bacteria $\cdot$ Grazing $\cdot$ Model $\cdot$ Mortality $\cdot$ Phage $\cdot$ Virus

\section{INTRODUCTION}

The potential influence of viruses on the dynamics of natural bacterial populations has been the subject of great interest over the past decade or so (see reviews by Thingstad et al. 1993, Bratbak et al. 1994, Suttle 1994). It has been suggested that viral infection can contribute significantly to overall bacterial mortality in many marine and freshwater environments (e.g. Proctor \& Fuhrman 1990, Heldal \& Bratbak 1991, Fuhrman

•E-mail: bbinder@uga.edu
\& Noble 1995, Hennes \& Simon 1995, Mathias et al. 1995, Weinbauer \& Peduzzi 1995, Steward et al. 1996, Weinbauer \& Höfle 1998). Support for this suggestion is often based on estimates of virally induced mortality calculated from the frequency of infected cells (FIC) within the bacterial populations of interest. Proctor \& Fuhrman (1990, see also Proctor et al. 1993) used a simple model to argue that the proportion of total bacterial mortality that is due to virally induced lysis (referred to here as the fractional mortality from viral lysis, FMVL) is equal to FIC multiplied by a factor of 2 . This 'factorof-two rule' has been widely used ever since, although it has not been rigorously examined (e.g. Suttle 1994, 
Fuhrman \& Noble 1995, Hennes \& Simon 1995, Mathias et al. 1995, Steward et al. 1996, Weinbauer \& Höfle 1998).

The rationale behind the factor-of-two rule is most concisely stated by Proctor et al. (1993): 'In this steady state model, we assume that bacterial growth rates are balanced by bacterial mortality rates, i.e., bacteria undergo binary fission and one daughter cell survives to divide again. Thus, if latent periods are similar in length to generation times, this suggests that a reasonable estimate of the percentage of total bacterial mortality attributable to virally induced lysis is two times the percent of bacteria which are phage-infected...' The explicit assumptions underlying this model, then, are (1) that bacterial populations are in steady state, and (2) that the latent period is approximately equal to the generation time of uninfected bacteria. Additionally, the model makes the implicit assumption (3) that all infoctcd cells die from lysis - i.e. that infected celis are not grazed.

In practice, the factor-of-two rule is generally applied to FIC values calculated from measurements of the proportion of bacterial cells containing recognizable virus particles (frequency of visibly infected cells, FVIC), combined with estimates of the fraction of the lytic cycle during which such particles are likely to be visible. Specifically, this calculation takes FVIC/FIC to be equal to this latter fraction (Proctor et al. 1993). As will be shown later, this calculation itself implicitly relies on assumption 3 , above.

In this paper, a simple mathematical model is used to test the validity of the factor-of-two rule, to develop a

Table 1. Abbreviations and symbols used in the text and the model. Units indicated in parentheses; where excluded, quantity is dimensionless

\begin{tabular}{|c|c|}
\hline FIC & Frequency of infected cells $\left[\equiv N_{1} /\left(N_{i}+N_{u}\right)\right]$ \\
\hline FVIC & Frequency of visibly infected cells \\
\hline FMVL & Fractional mortality from viral lysis $\left[\equiv L /\left(L+G_{1}+G_{u}\right)\right]$ \\
\hline$N_{i}$ & Abundance of infected cells (cells $\mathrm{ml}^{-1}$ ) \\
\hline$N_{u}$ & Abundance of uninfected cells (cells $\mathrm{ml}^{-1}$ ) \\
\hline$N_{\mathrm{v}}$ & Abundance of visibly infected cells (cells $\mathrm{ml}^{-1}$ ) \\
\hline$D$ & Cell division rate (absolute) (cells $\mathrm{ml}^{-1} \mathrm{~d}^{-1}$ ) \\
\hline$I$ & Infection (or induction) rate (absolute) (cells $\mathrm{ml}^{-1} \mathrm{~d}^{-1}$ ) \\
\hline$L$ & Rate of viral lysis (absolute) (cells $\mathrm{ml}^{-1} \mathrm{~d}^{-1}$ ) \\
\hline$G_{1}$ & Grazing mortality rate, infected cells (absolute) (cells $\mathrm{ml}^{-1} \mathrm{~d}^{-1}$ ) \\
\hline$G_{12}$ & Grazing mortality rate, uninfected cells (absolute) (cells $\mathrm{ml}^{-1} \mathrm{~d}^{-1}$ ) \\
\hline$\mu$ & Specific rate of cell division $\left(\mathrm{d}^{-1}\right)$ \\
\hline$g_{1}$ & Specific rate of grazing mortality, infected cells $\left(\mathrm{d}^{-1}\right)$ \\
\hline$g_{u}$ & Specific rate of grazing mortality, uninfected cells $\left(\mathrm{d}^{-1}\right)$ \\
\hline$t_{\mathrm{L}}$ & Latent period (d) \\
\hline$t_{\mathrm{G}}$ & Generation time of uninfected cells $(\equiv \ln (2) / \mu)(d)$ \\
\hline$\Phi(a)$ & Frequency of infected cells of age $a$ (cells $\left.\mathrm{d}^{-1}\right)$ \\
\hline$\alpha$ & Ratio of infected to uninfected specific grazing mortality rates, $g_{1} / g_{u}$ \\
\hline$\beta$ & Ratio of uninfected grazing rate to division rate, $g_{u} / \mu$ \\
\hline$y$ & Ratio of latent period to generation time, $t_{L} / t_{G}$ \\
\hline$\varepsilon$ & Fraction of $t_{L}$ elapsed prior to appearance of intracellular viral particle \\
\hline
\end{tabular}

more accurate description of the relationships between FVIC, FIC, and FMVL, and to examine the sensitivity of these relationships to violations of assumptions 2 and 3 , above. Other recent models involving aquatic bacteriophages have been directed toward exploring the implications of viral activity on nutrient cycling in microbial food webs or toward understanding the biological conditions required to sustain viral populations; none has addressed the relationships between FVIC, FIC, and FMVL (Murray \& Eldridge 1994, Blackburn et al. 1996, Beretta \& Kuang 1998)

\section{RESULTS}

\section{The model}

A simple box model of a bacterial population subject to virai infection is illustrated in Fig. 1; Table 1 summarizes the terms describing this model. The model consists of infected and uninfected bacterial sub-populations, present in abundances $N_{\mathrm{t}}$ and $N_{\mathrm{u}}$ respectively. The only source of new cells to the population is cell division, $D$, which occurs in uninfected cells exclusively. Thus,

$$
D=\mu N_{\mathrm{u}}
$$

where $\mu$ is defined as the specific growth rate of the population $\left(\mathrm{d}^{-1}\right)$. Infected cells are produced from uninfected cells via viral infection $(I)$. For the purpose of this model, all infections are assumed to be lytic this makes sense in the context of establishing the relationship between FIC and mortality, as lysogenic infection would not be counted in estimates of FIC for lead to lysis) until induction has occurred.

Bacterial loss terms include viral lysis of infected cells $(L)$, and grazing mortality for both infected and uninfected populations $\left(G_{\mathrm{i}}\right.$ and $G_{\mathrm{u}}$, respectively). Expressions for $L$ will be developed below. Grazing mortality is expressed as the product of prey concentration and a specific grazing mortality rate, so that

$G_{\mathrm{i}}=g_{\mathrm{i}} N_{\mathrm{i}}$ and $G_{\mathrm{u}}=g_{\mathrm{u}} \cdot N_{\mathrm{u}}$

where $g_{\mathrm{i}}$ and $g_{\mathrm{u}}$ are the specific grazing mortality rates $\left(\mathrm{d}^{-1}\right)$ for infected and uninfected cells, respectively. Although the model does not explicitly include any other loss terms, such terms may 


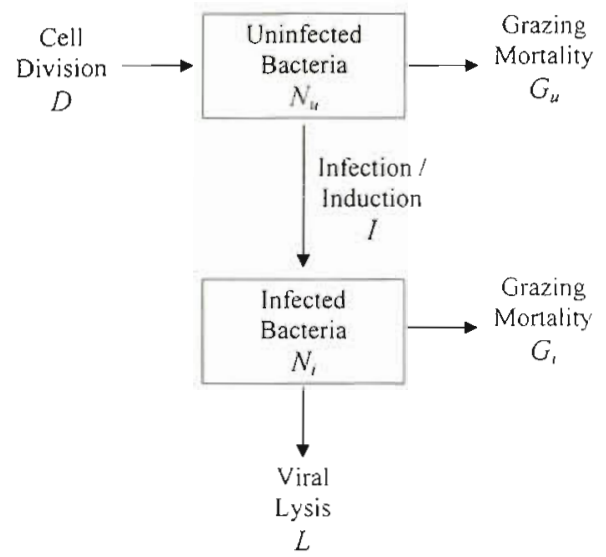

Fig. 1. Schematic of the model. See Table 1 for definitions

be aggregated with grazing mortality. Note that, in this steady-state model, no particular functional relationship between specific grazing mortality rate and prey concentration is assumed.

\section{Virally induced mortality}

At steady state, total bacterial growth must balance total bacterial mortality. 'Thus,

$$
D=G_{u}+G_{i}+L
$$

and therefore,

$$
\mu N_{\mathrm{u}}=g_{\mathrm{u}} N_{\mathrm{u}}+g_{\mathrm{i}} N_{\mathrm{i}}+L
$$

Rearranging terms,

$$
L=\mu N_{u}-\left(g_{u} N_{u}+g_{1} N_{1}\right)
$$

Recall that FMVL $=L /$ total mortality. Since total mortality equals total growth,

$$
\text { FMVL }=\frac{L}{\mu N_{u}}
$$

Then, from Eqs. (5) \& (6),

$$
\text { FMVL }=1-\beta\left(1+\alpha \frac{N_{1}}{N_{\mathrm{u}}}\right)
$$

where $\alpha=g_{\mathrm{i}} / g_{\mathrm{u}}$ and $\beta=g_{\mathrm{u}} / \mu$. Note that $N_{\mathrm{i}} / N_{\mathrm{u}}$ is itself a function of $\alpha$ and $\beta$ (see below).

These expressions for $L$ and FMVL are based on the balance between growth and loss terms that is mandated by steady-state conditions. Another set of expressions can be derived from the expected relationship between the rate of viral lysis and the size and composition of the infected population. Unlike cell division and grazing mortality, virally induced mortality is not strictly proportional to the total abundance of infected cells, but rather depends upon the number of cells that have been infected for a time equal to the latency period $\left(t_{L}\right)$. This number can be derived from $N_{\mathrm{i}}$ if the frequency distribution of infection-age among the infected sub-population is known. 'Infection-age' is used here to refer to the amount of time that has passed since a given cell was first infected.

We will express the infection-age distribution, $\Phi(a)$, in terms of the frequency of cells (cells time ${ }^{-1}$ ) of a given infection-age, $a$. The size of the age a 'cohort' is then given by $\Phi(a)$ da. If we consider the cohort of cells that have just been infected $(a=0)$, and experience a constant specific grazing rate, $g_{i}$, the number of cells in that cohort surviving until infection-age, $a$, is given by

$$
\Phi(a) \mathrm{d} a=\Phi(0) \mathrm{e}^{-g_{1} a} \mathrm{~d} a \quad \text { for } 0 \leq a \leq t_{\mathrm{L}}
$$

Those cells that survive until $a=t_{\mathrm{L}}$ are lysed. Because under steady-state conditions $\Phi(0)$ remains constant and all cohorts of cells experience identical survivorship, Eq. (8) describes the steady-state infection-age distribution of the infected sub-population. Furthermore, $\Phi(0)$ and $\Phi\left(t_{\mathrm{L}}\right)$ are equal to the steady-state rate of infection and lysis, respectively. Fig. 2 illustrates the infection-age distribution of the infected sub-population for 3 different values of $g_{i}$. Note that when infected cells are not grazed $\left(g_{3}=0\right)$, the distribution reduces to the form $\Phi(a)=\Phi(0)$, meaning that all infected cells survive until lysis. At the other extreme, when infected cells are grazed very strongly, $\Phi\left(t_{\mathrm{L}}\right)$ approaches 0 and very few cells die from lysis.

Integration of the infection-age distribution yields the size of the infected sub-population, and therefore by definition equals $N_{1}$. This relationship can be used to solve for $\Phi\left(t_{\mathrm{L}}\right)(=L)$ in terms of $N_{\mathrm{i}}$ :

$$
N_{1}=\int_{0}^{t_{\mathrm{L}}} \Phi(0) \mathrm{e}^{-g_{1} a} \mathrm{~d} a=\frac{\Phi(0)}{g_{\mathrm{i}}}\left(1-\mathrm{e}^{-g_{1} t_{\mathrm{L}}}\right)
$$

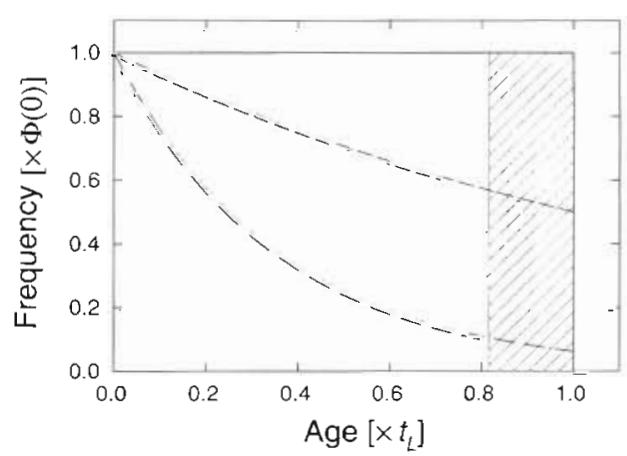

Fig. 2. Infection-age distributions of the infected sub-population of bacteria when $g_{i}=0$ (solid line), $\ln (2)$ (broken line), and $4 \times \ln (2)$ (dotted broken line). $y$-axis is cell frequency, normalized to age-0 frequency; $x$-axis is infection-age (i.e. time since initial infection), normalized to the length of the latent period. Hatched area: portion of the latent period during which viral particles are recognizable, assuming that $\varepsilon=0.816$ (see text) 
Rearranging terms and combining with Eq. (8),

$$
L=\Phi\left(t_{L}\right)=\frac{N_{\mathrm{i}} g_{\mathrm{I}}}{\mathrm{e}^{g_{\mathrm{L}} t_{L}}-1} \quad \text { for } g_{\mathrm{i}}>0
$$

Evaluating this expression as $g_{\mathrm{i}} \rightarrow 0$ yields

$$
L=\frac{N_{\mathrm{i}}}{t_{\mathrm{L}}} \quad \text { for } g_{\mathrm{i}}=0
$$

For convenience, these equations can be expressed in terms of $g_{u}$ and $\mu$ :

$$
L= \begin{cases}\frac{\alpha N_{\mathrm{i}} g_{u}}{2^{\alpha \beta f}-1} & \text { for } \alpha \beta>0 \\ \frac{\mu N_{i}}{\gamma \ln (2)} & \text { for } \alpha \beta=0\end{cases}
$$

where $\gamma=t_{\mathrm{L}} / t_{\mathrm{G}}$ and $t_{\mathrm{G}}=$ generation time $=\ln (2) / \mu$. Combining Eqs. (́) \& (12),

$$
\text { FMVL }= \begin{cases}\frac{\alpha \beta}{2^{\alpha \beta \gamma}-1}\left(\frac{N_{\mathrm{i}}}{N_{\mathrm{u}}}\right) & \text { for } \alpha \beta>0 \\ \frac{1}{\gamma \ln (2)}\left(\frac{N_{\mathrm{i}}}{N_{\mathrm{u}}}\right) & \text { for } \alpha \beta=0\end{cases}
$$

Eqs. (7) \& (13) are independent expressions that can be solved simultaneously for $N_{\mathrm{i}} / N_{\mathrm{u}}$, for given values of $\alpha$, $\beta$, and $\gamma$

$$
\frac{N_{1}}{N_{u}}= \begin{cases}\frac{1-\beta}{\alpha \beta}\left(1-2^{-\alpha \beta \gamma}\right) & \text { for } \alpha \beta>0 \\ \gamma \ln (2)(1-\beta) & \text { for } \alpha \beta=0\end{cases}
$$

Together, Eqs. (7) \& (14) fully describe the relationship between FMVL and $N_{\mathrm{i}} / N_{\mathrm{u}}$. Because experimental studies usually express the frequency of viral infection in terms of FIC $\left[=N_{\mathrm{i}} /\left(N_{1}+N_{\mathrm{u}}\right)\right]$ rather than $N_{\mathrm{i}} / N_{\mathrm{u}}$, these equations can be recast as

$$
\text { FMVL }=1-\beta\left(1+\alpha \frac{\text { FIC }}{1-\text { FIC }}\right)
$$

and

$$
\text { FIC }= \begin{cases}{\left[\frac{\alpha \beta}{(1-\beta)\left(1-2^{-\alpha \beta \gamma}\right)}+1\right]^{-1}} & \text { for } \alpha \beta>0 \\ {\left[\frac{1}{\gamma(1-\beta) \ln (2)}+1\right]^{-1}} & \text { for } \alpha \beta=0\end{cases}
$$

In the discussion that follows, FMVL and FIC are taken as dependent variables in the system, $\alpha$ and $\gamma$ are considered parameters that describe biological characteristics of the system, and $\beta$ is taken as the independent variable. In the real world, $\beta$ reflects the balance between grazing and infection as loss terms from the uninfected population (since the sum of these 2 terms must balance the growth rate at steady state) (Fig. 1).

The analytical derivation of FMVL as a function of FIC (and $\alpha$ and $\gamma$ ) from Eqs. (15) \& (16) is difficult, except in the special case when $\alpha=0$ (see below). However, by calculating both FMVL and FIC for a range of $\beta$, the relationship between FIC and FMVL under various combinations of $\alpha$ and $\gamma$ can be examined (Figs. 3 \& 4; see also Fig. 6). Numerical solutions for cases of particular interest are easily obtained (see 'Discussion').

\section{Case 1: infected cells not grazed; latent period = generation time}

The derivation of the factor-of-two rule assumes that the latent period is approximately equal to the generation time and that infected cells are not grazed. Under these conditions, Eqs. (15) \& (16b) can be combined to yield

$$
\text { FMVL }=\frac{1}{\ln (2)}\left(\frac{\text { FIC }}{1-\text { FIC }}\right) \text { for } \alpha=0 \text { and } \gamma=1
$$

Clearly, then, the factor-of-two rule (which states that FMVL $=2$ FIC) is incorrect, even given the restrictive assumptions upon which it was based. Nevertheless, under these assumptions (including the absence of grazing on infected cells), the error introduced by the factor-of-two rule is relatively modest, ranging from an $18 \%$ underestimate at the highest possible FIC $(=0.41$, see 'Discussion') to a $\sim 38 \%$ overestimate as FIC approaches 0 (Fig. 3, solid line).

\section{Case 2: infected cells grazed; latent period = generation time}

The relationship between FMVL and FIC changes when we introduce non-zero grazing on infected cells Grazing mortality provides an alternate fate for infected cells, thus decreasing FMVL for a given value of FIC, and increasing the error of the factor-of-two rule, relative to the no-grazing case (Fig. 3). This effect increases with increasing grazing rate on infected cells $(\alpha)$, and with decreasing FIC. Thus, if the specific grazing rate for uninfected and infected cells is equal ( $\alpha=$ 1), the factor-of-two rule overstates FMVL by a factor of 2 at low FIC; if $\alpha$ is increased to 2 (implying preferential grazing on infected cells), that error at low FIC increases to a factor of 3 . For higher values of FIC (> 0.3), however, the error remains small. 


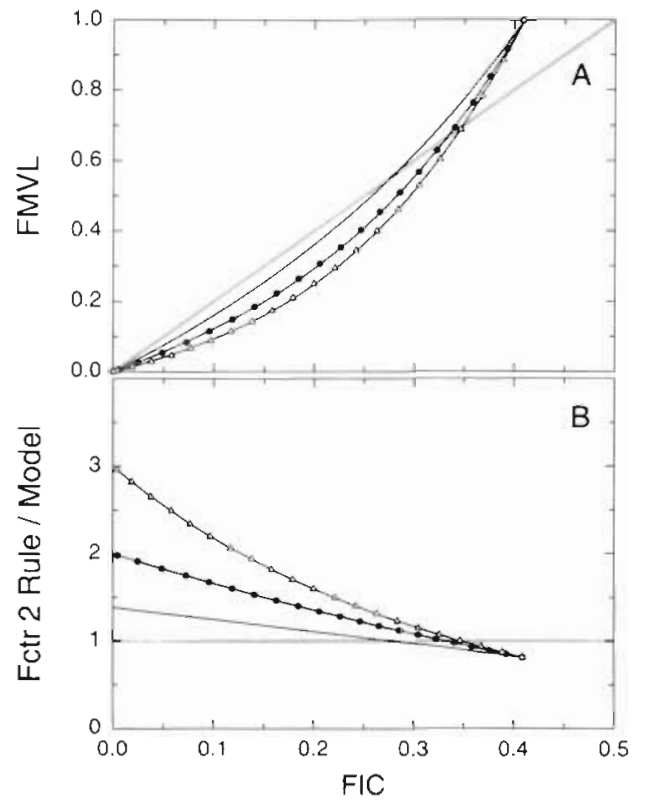

Fig. 3. Relationship between FMVL and FIC predicted by the model for $\alpha=0$ (solid line), $1(\bullet$ ), and $2(\Delta) ; \gamma=1$ in all cases. For the first case, line shown is the analytical solution of the model (Eq. 17); for the other cases, symbols are exact solutions for various values of $\beta$, and lines are best fits of the form FMVL $=\left(F I C+a \mathrm{FIC}^{2}\right) /(b+c F I C)$. Double line: factor-of-two rule prediction. (A) FMVL versus FIC. (B) Ratio of the factorof-two rule prediction and the model prediction

\section{Case 3: effect of varying latent period}

The model allows us to test the sensitivity of the calculated FMVL to deviations from the assumption that $t_{\mathrm{G}}=t_{\mathrm{L}}$ (i.e. that $\gamma=1$ ). Fig. 4 shows that calculated FMVL is in fact quite sensitive to $\gamma$. Thus, in the example shown $(\alpha=1)$, a $50 \%$ increase in $\gamma$ leads to a dramatic reduction in FMVL for a given FIC, and a corresponding increase in the error associated with both the factor-of-two rule calculation and the $\gamma=1$ case of the present model. Conversely, a $50 \%$ decrease in $\gamma$ results in a great increase in FMVL, and corresponding underestimates by the factor-of-two rule and the $\gamma=1$ case of the model.

\section{Calculating FIC from FVIC}

The preceding analysis describes the influence of $\alpha$ and $\gamma$ on the relationship between FMVL and true FIC. In practice, however, FIC is derived from measured values of FVIC, and the relationship between these 2 quantities is itself dependent on $\alpha$ and $\gamma$ (see below).

Given that by definition FIC $=N_{\mathrm{i}} /\left(N_{\mathrm{i}}+N_{\mathrm{u}}\right)$ and FVIC $=N_{v} /\left(N_{1}+N_{u}\right)$, (where $N_{v}=$ number of visibly infected cells), it follows that

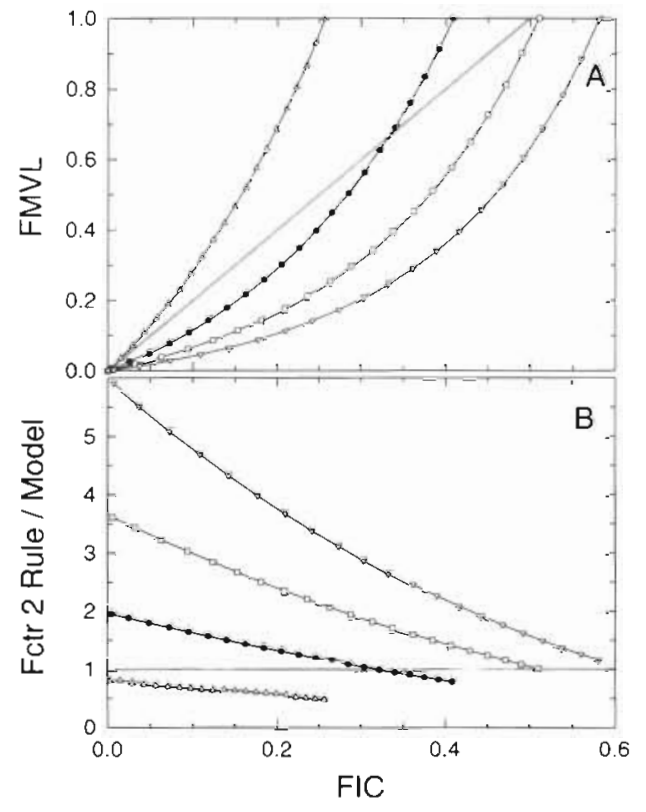

Fig. 4. Relationship between FMVL and FIC predicted by the model for $\gamma=0.5(\Delta), 1(\bullet), 1.5(\square)$, and $2(\nabla) ; \alpha=1$ in all cases. Lines as in Fig. 3. Note change of scales

$$
\mathrm{FIC}=\left(N_{\mathrm{i}} / N_{\mathrm{v}}\right) \text { FVIC }
$$

Assuming that recognizable viral particles appear in infected cells only after some fraction $(\varepsilon)$ of the latent period has elapsed, $N_{v}$ can be calculated as the integral of the infection-age distribution (as defined in Eq. 8) between $\mathrm{a}=\varepsilon t_{\mathrm{L}}$ and $t_{\mathrm{L}}$ :

$$
N_{\mathrm{v}}=\int_{\mathcal{E} t_{L}}^{t_{L}} \Phi(0) \mathrm{e}^{-g_{1} a} \mathrm{~d} a=\frac{\Phi(0)}{g_{\mathrm{i}}}\left(\mathrm{e}^{-g_{1} \varepsilon t_{L}}-\mathrm{e}^{-g_{1} t_{\mathrm{L}}}\right)
$$

Combining Eqs. (9) \& (19)

$$
N_{\mathrm{b}} / N_{\mathrm{y}}=\frac{\mathrm{e}^{g_{1} t_{\mathrm{L}}}-1}{\mathrm{e}^{g_{\mathrm{I}} t_{\mathrm{L}}(1-\varepsilon)}-1}
$$

Thus, just as grazing mortality reduces the proportion of infected cells that survive long enough to lyse, so does it reduce the proportion of cells that survive long enough to contain visible viral particles (Fig. 2).

Eq. (20) can be expressed in terms $\alpha, \beta$, and $\gamma$ :

$$
N_{\mathrm{i}} / N_{\mathrm{v}}=\frac{2^{\alpha \beta \gamma}-1}{2^{\alpha \beta \gamma(1-\varepsilon)}-1} \quad \text { for } \alpha \beta \gamma>0
$$

Evaluating this expression as $\alpha \beta \gamma \rightarrow 0$ yields

$$
N_{1} / N_{\mathrm{v}}=1 /(1-\varepsilon) \quad \text { for } \alpha \beta \gamma=0
$$

Finally, combining Eqs. (18) \& (21),

$$
\text { FIC }= \begin{cases}\text { FVIC }\left[\frac{2^{\alpha \beta \gamma}-1}{2^{\alpha \beta \beta \gamma(1-\varepsilon)}-1}\right] & \text { for } \alpha \beta \gamma>0 \\ \text { FVIC } /(1-\varepsilon) & \text { for } \alpha \beta \gamma=0\end{cases}
$$




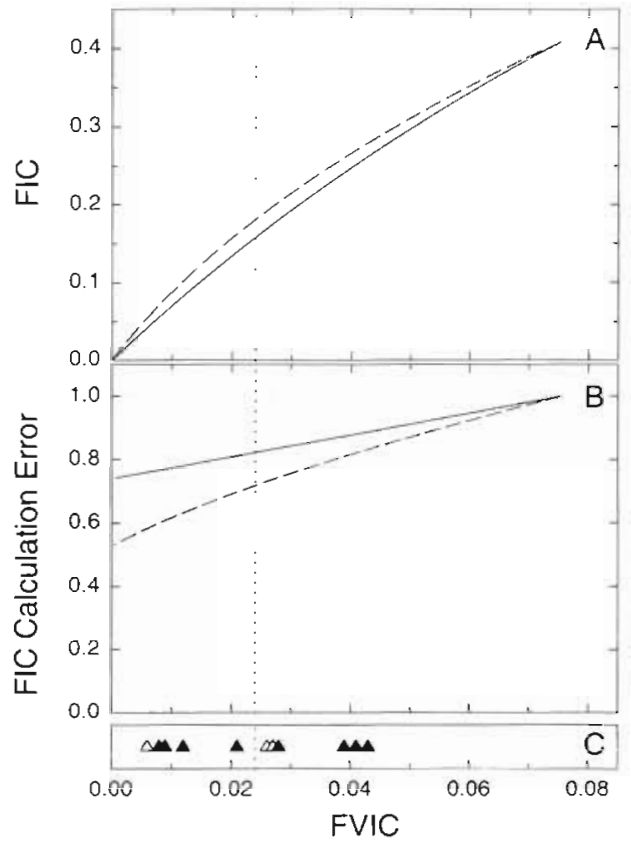

Fig. 5. Relationship between FIC and FVIC predicted by the model for $\alpha=0$ (dotted line), 1 (solid line), and 2 (broken line); $\gamma=1$ and $\varepsilon=0.816$ in all cases. (A) FIC versus FVIC. (B) Ratio of traditionally calculated FIC (using a conversion factor of 5.42) and FIC predicted by the model. (C) Literature values of mean observed FVIC in a number of marine ( $\mathbf{A}$ ) and freshwater $(\Delta)$ environments, as summarized in Table 2 . Vertical dotted line corresponds to the average of these ecosystemspecific means. See Table 2 for calculation details

Note that Eq. (22b) is identical to the relationship routinely employed to calculate FIC from FVIC (Proctor et al. 1993), but that it is only correct when grazing mortality among infected cells $=0$ (i.e. when $\alpha=0$ ). As $\alpha$ increases above 0, FIC calculated from Eq. (22b) progressively underestimates true FIC (Fig. 5). At the average observed FVIC of $2.4 \%$ (Table 2), and assuming $\alpha=\gamma=1$, Eq. (22b) underestimates true FIC by approximately $17 \%$ (Fig. 5b).

Therefore, while non-zero grazing mortality among infected cells (non-zero $\alpha$ ) results in an overestimate of FMVL (as calculated from the $\alpha=0$ case of the model) for a given value of FIC, at the same time it results in an underestimate of FIC (as calculated from Eq. 22b) for a given value of FVIC. These 2 errors tend to balance each other, such that the relationship between FMVL and FVIC is to a first approximation insensitive to $\alpha$ (Fig. 6). This allows us to use the analytically simple $\alpha=0$ case of Eqs. (15), (16) \& (22) to derive a general expression for the relationship between FMVL and FVIC:

$$
\text { FMVL } \cong \frac{1}{\gamma \ln (2)}\left(\frac{F V I C}{(1-\varepsilon)-F V I C}\right)
$$

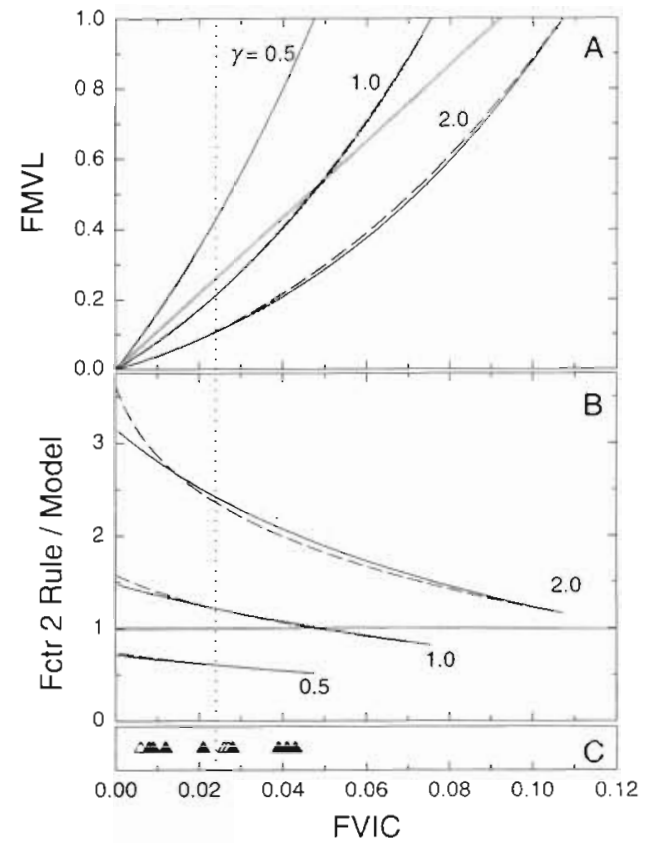

Fig. 6. Relationship between FMVL and FVIC predicted by the model, for $\gamma=0.5,1$, and 2, as indicated. Lines show prediction for $\alpha=0,1$, and 2, as in Fig. $5_{i} \varepsilon=0.816$ in all cases. Double lines: factor-of-two rule prediction. (A) FMVL versus FVIC. (B) Ratio of the factor-of-two rule prediction and the model prediction. (C) Summarized literature values of FVIC, as in Fig. 5

More precise solutions for specific values of $\alpha, \gamma$, and $\varepsilon$ can be derived numerically (see below).

\section{DISCUSSION}

\section{The factor-of-two rule}

The model presented here indicates that the widely used factor-of-two rule for relating FMVL to FIC is in error. Under the steady-state conditions assumed for the derivation of that rule (no grazing on infected cells, and $t_{\mathrm{L}}=t_{\mathrm{G}}$ ), actual FMVL is $>2$ FIC when FIC $>33 \%$, and $<2$ FIC when FIC $<33 \%$ (Fig. 3). These deviations are the result of 2 opposing problems in the original derivation of the factor-of-two rule. First, the derivation fails to take into account the continuous nature of growth and mortality of cells in a population. The exponential growth equation dictates that a population of size $N$ that is in balanced growth must experience a total mortality of $N$ $\ln (2)(\sim 0.69 N)$ over the course of 1 generation if $N$ is to remain constant. In contrast, Proctor \& Fuhrman (1990) assumed that the total mortality of such a population = $0.5 \mathrm{~N}$. Thus, by underestimating total mortality in steady state, the factor-of-two rule would tend to overestimate the relative contribution of viral lysis to that mortality 
Table 2. Summary of published FVIC values for marine and freshwater environments, and FMVL values calculated therefrom using 3 different models. Overall mean value for each environment is reported, without regard to differences in depth or season. Only studies using electron microscopy to measure FVIC are included; no correction has been applied for thin section versus whole cell observations. Calculation details are listed in the footnotes. Values in bold correspond to recommended model (see 'Discussion')

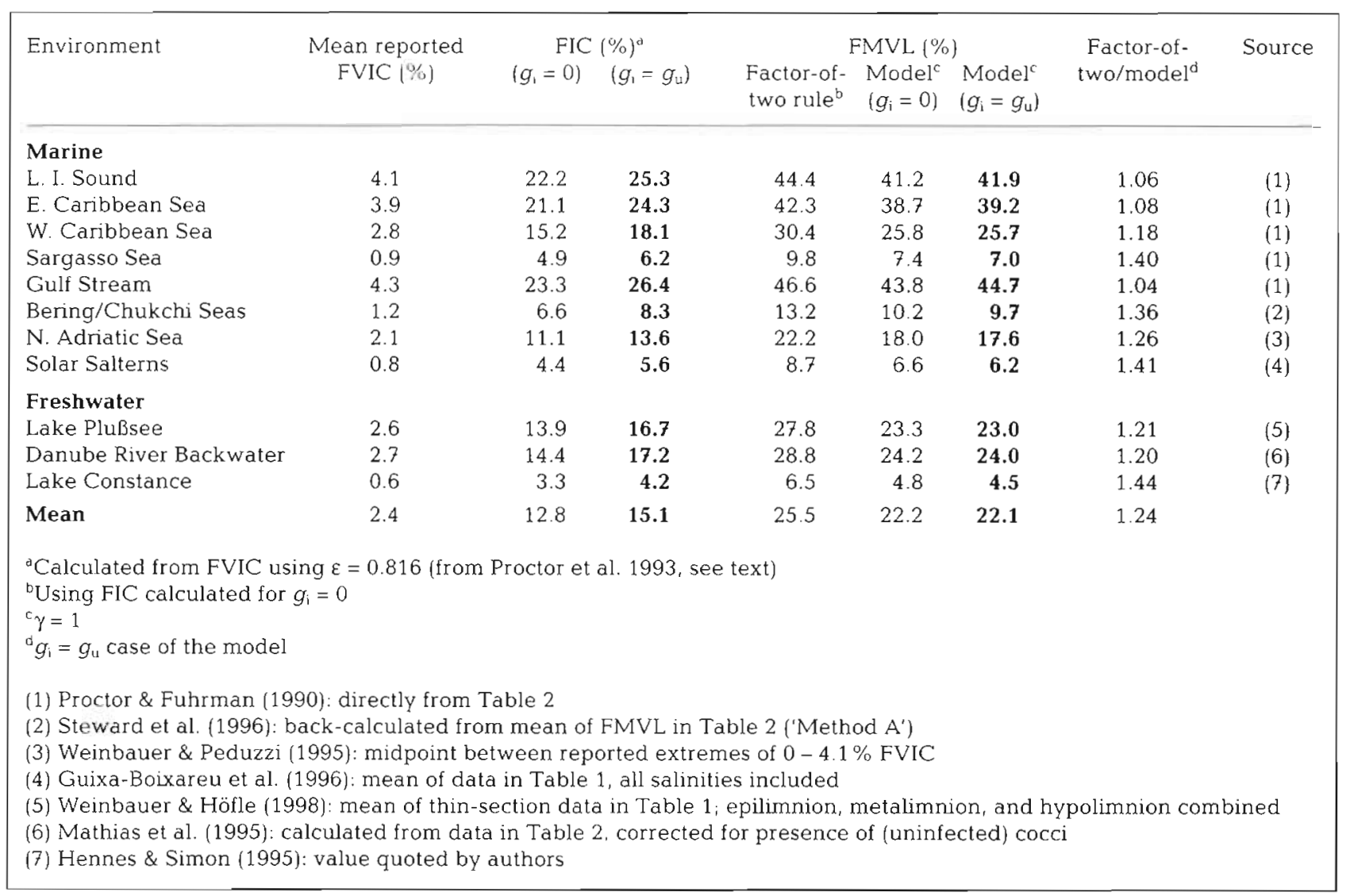

On the other hand, just as cell division and mortality are continuous processes, so is viral infection. Again, the factor-of-two rule does not take this into account. Over the course of 1 generation, there is a certain probability that a cell will be infected before it divides, and will thus be prevented from contributing further to the growth of the population. This removal of cells from the actively dividing sub-population (but not from the population as a whole) decreases the effective growth rate of the population at large, which in turn decreases the overall mortality rate required to maintain steady state, and therefore increases FMVL for a given rate of viral lysis. This explains why viral lysis accounts for $100 \%$ of mortality (i.e. FMVL reaches 1.0 ) at values of $\mathrm{FIC}<50 \%$ (as long as $\gamma=1$ ) (Fig. 3). The model indicates that, for values of FIC $>\sim 0.3$, the depression of effective overall growth rate by viral infection is strong enough to result in somewhat higher FMVL than predicted by the factor-of-two rule (maximum underestimate of the rule $\cong 18 \%$ ); at values below this level, this effect becomes progressively less important, and the factor-of-two rule overestimates FMVL (maximum overestimate $\cong 38 \%$ ).

\section{Incorporating grazing}

Eq. (9) gives the correct description of the relationship between FMVL and FIC in the absence of grazing on infected cells (and given that $t_{\mathrm{L}}=t_{\mathrm{G}}$ ). However, this condition may be unrealistic for natural systems (see below). If grazing mortality does occur among infected cells, it will reduce the fraction of those cells that survive long enough to lyse, and therefore reduce FMVL for a given value of FIC (Figs. $2 \& 3$ ). The partitioning of the mortality of infected cells between grazing and lysis is not just a mathematical nicety, but rather has serious implications for the flow of energy and material through the microbial food web. If infected cells are grazed before they lyse their biomass is accessible to higher trophic levels to the same extent as uninfected bacterial biomass is; if those cells instead survive long 
enough to lyse, a large proportion of that biomass instead presumably enters the DOM pool (Thingstad et al. 1993, Bratbak et al. 1994). Note that, for all values of $\alpha$ (and assuming $\gamma=1$ ), the maximum possible FIC is 0.41 (Fig. 3). Measured FIC values greater than 0.41 must be the result of either non-steady-state conditions, values of $\gamma>1$ (see below), or errors in the estimation of FIC.

The assumption that infected cells are not grazed is implicit in the derivation of the factor-of-two rule (see 'Introduction') and in any other case in which all infected cells are assumed to ultimately lyse (e.g. Guixa-Boixareu et al. 1996, Beretta \& Kuang 1998). However, there are to my knowledge no data supporting the contention that infected cells are not consumed by bacterivores; in fact it has been suggested that such cells might be consumed preferentially by grazers (Weinbauer \& Peduzzi 1995). In the absence of evidence to the contrary, it seems that the most appropriate a priori assumption is that infected and uninfected cells are grazed at equal specific rates (i.e. $\alpha=1$ ). The relationship between FMVL and FIC under this condition (and assuming $\gamma=1$ ) is shown in Fig. 3 (closed symbols), and is very well approximated by the equation:

$$
\mathrm{FMVL} \cong \frac{\mathrm{FIC}+0.6 \mathrm{FIC}^{2}}{1-1.2 \mathrm{FIC}}
$$

Given accurate measurements of FIC, this equation provides better estimates of FMVL than does the factor-of-two rule.

\section{Relationship between FMVL and FVIC}

In infected cells, recognizable viral particles are present only in relatively late stages of the lytic cycle. Because grazing mortality among these cells skews the infection-age distribution of the population towards more recently infected cells (Fig. 2), it also lowers the proportion of those cells that are visibly infected. Thus, in addition to requiring knowledge about the fraction of the latent period during which recognizable viral particles are not present $(\varepsilon)$, accurate calculation of FIC from FVIC also requires an assumption about the magnitude of grazing mortality in the infected sub-population (Eq. 22, Fig. 5). Eqs. (16) \& (22) together define the relationship between FIC and FVIC at steady state. For the specific case that $\alpha=\gamma=1$ and $\varepsilon=0.186$ (see below), this relationship is well described by:

$$
\mathrm{FIC} \cong 7.1 \mathrm{FVIC}-22.5 \mathrm{FVIC}^{2}
$$

Using FIC values derived from this formula in combination with Eq. (24) would provide the most accurate estimate of FMVL, absent specific data concerning $\alpha, \gamma$, or $\varepsilon$. Eq. (23) represents a somewhat less precise, but more general (and informative), solution for the relationship between FMVL and FVIC.

The effect of grazing mortality on the relationship between FVIC and FIC documented here has not been recognized previously. Because this effect is largely balanced by the opposite effect on FMVL calculated from FIC (see 'Results'), in the context of estimating FMVL it is to some extent moot. Nevertheless, under those circumstances when the value of FIC itself is of interest, this effect should be taken into account.

\section{Implications for interpretation of field data}

In order to put these results into an ecologically relevant context, published values of FVIC from a range of aquatic environments are summarized in Table 2. Within each study area, individual determinations of FVIC generally varied very widely. Furthermore, many of these studies examined samples encompassing a range of depths, seasons, temperatures, and/or salinities. The range of values shown here (Table 2 , Figs. $5 \& 6$ ), each of which represents the average for a particular region or study area irrespective of these other variables, is therefore by no means indicative of the overall range of observed FVIC values in aquatic environments. Nevertheless, these averages certainly are indicative of typically observed FVIC values, and as such provide a useful framework for considering model predictions.

Average observed FVIC in the studies tabulated in Table 2 was $2.4 \%$. Assuming that steady state prevails, that infected and uninfected cells are grazed with equal efficiency, and that the latent period is equivalent to the generation time of uninfected cells, this corresponds to an average true FIC of $15.1 \%$. Under these assumptions, the model suggests that viral lysis is generally responsible for between 4.5 and $45 \%$ (mean = $22 \%$ ) of overall bacterial mortality in these environments. Over the observed range of FVIC, the factor-oftwo rule overestimates FMVL by an average of $24 \%$ (range 4 to $44 \%$ ) (Fig. 6).

The uncertainty associated with estimates of FIC in natural populations is quite large. In addition to the wide range of observed values of FVIC mentioned above, there is considerable uncertainty in the calculation of FIC from these observed values. As discussed previously, this conversion is dependent upon the fraction of the lytic cycle during which intracellular virus particles are recognizable $(1-\varepsilon)$. Proctor et al. (1993) estimated this fraction to be between 0.14 and 0.27 , based on studies with 2 marine Vibrio isolates. These values lead to estimated conversion factors $[1 /(1-\varepsilon)]$ of 
7.14 and 3.70 , respectively. Investigators typically use the mean of these values (5.42) for calculating FIC from FVIC (the corresponding value of $\varepsilon, 0.816$, is used here in Table 2, as well), but recognize that the uncertainty in this conversion factor is considerable. Examination of Eq. (23) indicates that uncertainty in $(1-\varepsilon)$ translates approximately proportionately to uncertainty in calculated FMVL, in the range of FVIC and $\varepsilon$ values typically observed.

Considerable uncertainty is also associated with the assumption that the latent period in infected cells equals the generation time of uninfected cells (i.e. that $\gamma=1$ ). The model shows that calculated values of FMVL are quite sensitive to deviations from this value (Figs. $4 \& 6$ ). This sensitivity is the result of changes both in the effective rate at which the infected population progresses through the lytic cycle and in the length of time over which grazing extracts cells from that population. While the available data do indicate that on average $\gamma \cong 1$, there is considerable variability among individual estimates of this parameter (Proctor et al. 1993, Guixa-Boixareu et al. 1996). In the data of Proctor et al. (1993), for example, $\gamma$ ranged between 0.6 and 1.5 for values of $t_{\mathrm{G}}>3 \mathrm{~h}$, and between 0.6 and 6 for $t_{\mathrm{G}}<3 \mathrm{~h}$ (mean $\cong 2$ in this latter case). Thus, the range of $\gamma$ illustrated in Figs. $4 \& 6$ is by no means extreme. At the average observed FVIC, FMVL = $42 \%, 22 \%$, and $11 \%$ for $\gamma=0.5,1$, and 2 , respectively (Fig. 6).

The sensitivity of FMVL to relatively small changes in $\varepsilon$ and $\gamma$ suggests that constraining these parameters with respect to environmental and biological variables would greatly improve the reliability of FVIC-based estimates of FMVL.

It is clear that estimates of fractional mortality from viral lysis based on measurements of the frequency of infected cells are subject to considerable uncertainty. It is not the intention of this paper to argue one way or the other regarding the value of such estimates in light of this uncertainty. Rather, the model presented here is intended to correct the previously unrecognized systematic error that has been incorporated into these estimates when they are made, to help point out avenues of future study that are likely to reduce the uncertainty in the approach, and to explore more fully the interaction between growth, grazing, and virally induced mortality in bacterial populations.

Editorial responsibility: Gunnar Bratbak, Bergen, Norway
Acknowledgements. This work was supported in part by a grant from the National Science Foundation (OCE-9711306). I am grateful to Jed Fuhrman for providing helpful comments on the manuscript. This paper is dedicated to the memory of Charles Abraham Binder.

\section{LITERATURE CITED}

Beretta $E$, Kuang $Y(1998)$ Modeling and analysis of a marine bacteriophage infection. Math Biosci 149:57-76

Blackburn N, Zweifel UL, Hagström \& (1996) Cycling of marine dissolved organic matter. II. A model analysis. Aquat Microb Ecol 11:79-90

Bratbak G, Thingstad F, Heldal M (1994) Viruses and the microbial loop. Microb Ecol 28:209-221.

Fuhrman JA, Noble RT (1995) Viruses and protists cause similar bacterial mortality in coastal seawater. Limnol Oceanogr 40: 1236-1242

Guixa-Boixareu N, Calderón-Paz JI Heldal M, Bratbak G, Pedrós-Alió C (1996) Viral lysis and bacterivory as prokaryotic loss factors along a salinity gradient. Aquat Microb Ecol 11:215-227

Heldal M, Bratbak G (1991) Production and decay of viruses in aquatic environments. Mar Ecol Prog Ser 72:205-212

Hennes KP, Simon M (1995) Significance of bacteriophages for controlling bacterioplankton growth in a mesotrophic lake. Appl Environ Microbiol 61:333-340

Mathias CB, Kirschner AKT, Velimirov B (1995) Seasonalvariations of virus abundance and viral control of the bacterial production in a backwater system of the Danube River. Appl Environ Microbiol 61:3734-3740

Murray AG, Eldridge PM (1994) Marine viral ecology: incorporation of bacteriophage into the microbial planktonic food-web paradigm. J Plankton Res 16:627-641

Proctor LM, Fuhrman JA (1990) Viral mortality of marinebacteria and cyanobacteria. Nature 343:60-62

Proctor LM, Okubo A, Fuhrman JA (1993) Calibrating estimates of phage-induced mortality in marine-bacteria ultrastructural studies of marine bacteriophage development from one-step growth experiments. Microb Ecol 25 : $161-182$

Steward GF, Smith DC, Azam F (1996) Abundance and production of bacteria and viruses in the Bering and Chukchi Seas. Mar Ecol Prog Ser 131:287-300

Suttle CA (1994) The significance of viruses to mortality in aquatic microbial communities. Microb Ecol 28:237-243

Thingstad TF, Heldal M, Bratbak G, Dundas I (1993) Are viruses important partners in pelagic food webs? Trends Ecol Evol 8:209-213

Weinbauer MG, Höfle MG (1998) Significance of viral lysis and flagellate grazing as factors controlling bacterioplankton production in a eutrophic lake. Appl Environ Microbiol 64:431-438

Weinbauer MG, Peduzzi P (1995) Significance of viruses versus heterotrophic nanoflagellates for controlling bacterial abundance in the northern Adriatic Sea. J Plankton Res $17: 1851-1856$

Submitted: September 14, 1998; Accepted: February 12, 1999 Proofs received from author(s): July 22, 1999 